\title{
Mediterranean diet and glycaemic load in relation to incidence of type 2 diabetes: results from the Greek cohort of the population-based European Prospective Investigation into Cancer and Nutrition (EPIC)
}

\author{
M. Rossi • F. Turati • P. Lagiou • D. Trichopoulos • \\ L. S. Augustin • C. La Vecchia • A. Trichopoulou \\ Received: 26 March 2013 / Accepted: 9 July 2013 / Published online: 22 August 2013 \\ (C) Springer-Verlag Berlin Heidelberg 2013
}

\begin{abstract}
Aims/hypothesis The role of diet in the prevention of diabetes remains uncertain. The aim of this study was to investigate two different dietary aspects, i.e. adherence to the Mediterranean diet and glycaemic load (GL), in relation to diabetes occurrence.

Methods We analysed data from the Greek cohort of the population-based European Prospective Investigation into Cancer and Nutrition (EPIC). From a total of 22,295 participants, actively followed for a median of 11.34 years, 2,330 cases of incident type 2 diabetes were recorded. All participants completed a validated, interviewer-administered semiquantitative food frequency questionnaire at enrolment. From
\end{abstract}

C. La Vecchia and A. Trichopoulou share senior co-authorship

Electronic supplementary material The online version of this article (doi:10.1007/s00125-013-3013-y) contains peer-reviewed but unedited supplementary material, which is available to authorised users.

M. Rossi $\cdot$ F. Turati $\cdot$ C. La Vecchia $(\bowtie)$

Department of Epidemiology, IRCCS - Istituto di Ricerche

Farmacologiche Mario Negri, via La Masa, 19, 20156 Milan, Italy

e-mail: lavecchia@marionegri.it

M. Rossi • C. La Vecchia

Department of Clinical Sciences and Community Health,

Università degli Studi di Milano, Milan, Italy

P. Lagiou $\cdot$ D. Trichopoulos

Department of Epidemiology, Harvard School of Public Health,

Boston, MA, USA

P. Lagiou $\cdot$ A. Trichopoulou

Department of Hygiene, Epidemiology and Medical Statistics,

School of Medicine, University of Athens, Athens, Greece

L. S. Augustin

Department of Nutritional Sciences, Faculty of Medicine,

University of Toronto, Toronto, ON, Canada this information, we calculated a ten point Mediterranean diet score (MDS), reflecting adherence to the traditional Mediterranean diet, as well as the dietary GL. We estimated HRs and the corresponding $95 \%$ CIs of diabetes using Cox proportional hazards regression models adjusted for potential confounders.

Results A higher MDS was inversely associated with diabetes risk (HR 0.88 [95\% CI 0.78, 0.99] for MDS $\geq 6$ vs MDS $\leq 3$ ). GL was positively associated with diabetes (HR 1.21 [95\% CI $1.05,1.40]$ for the highest vs the lowest GL quartile). A significant protection of about $20 \%$ was found for a diet with a high MDS and a low GL.

Conclusions/interpretation A low GL diet that also adequately adheres to the principles of the traditional Mediterranean diet may reduce the incidence of type 2 diabetes.

Keywords Cohort study Glycaemic load $\cdot$ Mediterranean diet · Risk · Type 2 diabetes

$\begin{array}{ll}\text { Abbreviations } \\ \text { EPIC } & \text { European Prospective Investigation into Cancer and } \\ & \text { Nutrition } \\ \text { GI } & \text { Glycaemic index } \\ \text { GL } & \text { Glycaemic load } \\ \text { MDS } & \text { Mediterranean diet score } \\ \text { MET } & \text { Metabolic equivalent task }\end{array}$

Introduction

The prevalence of diabetes mellitus, mostly type 2 , is increasing in many parts of the world [1]. Overweight and obesity are the key risk factors for this disease [2], whereas the role of diet composition-as well as other lifestyle factors-is still unquantified. Selected 'healthy eating' patterns - mainly 
characterised by a higher intake of fruit and vegetables - were associated with a lower risk of diabetes in various cohort studies [3, 4]. In particular, adherence to the Mediterranean diet was associated with a reduced risk of diabetes in three prospective studies [5-7] and one intervention study [8]. This suggests a detrimental effect of a diet rich in meat and a favourable role of a diet rich in olive oil, vegetables, fruit, nuts, cereals and legumes.

The rate of digestion and absorption of different carbohydrate sources may also influence diabetes risk. This is expressed in terms of a dietary index called the glycaemic index (GI) [9], which is an indicator of the carbohydrate's ability to raise blood glucose levels. It has been suggested that long-term consumption of high-GI foods increases insulin demand, promotes insulin resistance, impairs pancreatic beta cell function and eventually leads to type 2 diabetes [10-12]. Glycaemic load (GL), which is the product of the GI and the amount of carbohydrate in a food, has been subsequently proposed for epidemiological investigations in order to describe a diet according not only to the quality (i.e. GI) but also to the quantity of carbohydrates [10,11]. A higher GL has been associated with an increased risk of diabetes in several [13-16], although not in all [17-21], cohort studies.

In order to describe what people should eat to prevent diabetes, we have examined diet in terms of a Mediterranean diet score (MDS) and dietary GL and related them to type 2 diabetes incidence in a general population-based cohort in Greece, which is the Greek component of the European Prospective Investigation into Cancer and Nutrition (EPIC). We have focused on GL rather than GI because it is the product of GI (a food constant) and the amount of carbohydrate (a variable); however, we have also reported the overall findings for GI. Since adherence to both the Mediterranean diet and a low GL diet are in principle desirable [7, 13, 14], we have attempted to evaluate whether there is an empirically documentable benefit from their combination.

\section{Methods}

Study population A total of 28,572 participants from all over Greece were recruited between 1994 and 1999 as part of the Greek component of the EPIC study [22]. EPIC is a prospective cohort study investigating the role of biological, dietary, lifestyle and environmental factors in the aetiology of cancer and other chronic diseases. The study is conducted in 23 research centres in ten European countries [23]. All procedures are in accordance with the Helsinki Declaration, all participants have provided written informed consent, and the study protocol was approved by the ethics committees both at the International Agency for Research on Cancer (IARC) and at the University of Athens Medical School.
Data on diet A semi-quantitative food frequency questionnaire was used to assess usual intake of about 150 foods and beverages, as well as several complex recipes commonly used in Greece, during the year preceding enrolment. The questionnaire was administered in person by trained interviewers at recruitment. For each dietary item, participants were asked to report their frequency of consumption and portion size. The questionnaire was validated in terms of dietary records, including those that contributed to the computation of MDS, GL and GI [24].

Nutrient, ethanol and energy intakes were calculated using a food composition database modified to accommodate the particularities of the Greek diet [25].

MDS Conformity to the traditional Mediterranean diet was assessed through a score (i.e. MDS), relying on nine dietary components that capture the essence of the traditional Mediterranean diet [26]. A value of 0 or 1 was assigned to each component of the score as follows: for components frequently consumed in the traditional Mediterranean diet (i.e. vegetables, legumes, fruit and nuts, cereals, fish and seafood, as well a high ratio of monounsaturated to saturated lipids), participants whose consumption was above the sex-specific median were assigned a value of 1 ; otherwise, 0 . For components less frequently consumed in the traditional Mediterranean diet (dairy, as well as meat and meat products), participants whose consumption was at or below the sex-specific median were assigned a value of 1 ; otherwise, 0 . A value of 1 was also given to men consuming $10 \mathrm{~g}$ to less than $50 \mathrm{~g}$ of ethanol/day and to women consuming $5 \mathrm{~g}$ to less than $25 \mathrm{~g}$ of ethanol/day; otherwise, a value of 0 was assigned. Thus, the total MDS ranged from 0 (minimal adherence to the traditional Mediterranean diet) to 9 (maximal adherence to the traditional Mediterranean diet) (see electronic supplementary material [ESM] Table 1).

GL and GI The average daily GL was calculated for each study participant by adding up the products of the carbohydrate content per serving for each food, multiplied by the average number of servings of that food per day, multiplied by the food's GI [27, 28]. For each participant, we also computed average daily GI as GL divided by the total amount of available carbohydrate.

GI values of foods were assigned to items reported in the dietary questionnaires in a standardised manner as described in detail elsewhere [29]. In brief, GI values assigned to individual food items were obtained from the FosterPowell table [28], British values [30] and internet updates (www.glycemicindex.com), using glucose as the reference. Attention was paid to aspects that might influence the GI, including cooking method, preservation method, type of sugar and country-specific types of foods. For typically Greek recipes for which a GI had not been determined, we assigned the 
GI of the nearest comparable food, when available, or the GI of the food components or individual ingredients. Food items containing negligible or no carbohydrates (chiefly meat, fish, fats and eggs) were not assigned any values. Foods that most contributed to the GL estimate were bread (40\% among men and $31 \%$ among women), fruits (12\% and $14 \%$ ), pasta/rice/ other grains (9\% and $8 \%$ ), sugar and confectionary $(7 \%$ and $9 \%$, sweet buns/cakes/pies/biscuits (5\% and 9\%) and vegetables (5\% and 5\%) [29]. The correlation coefficient between dietary GL and total carbohydrates was 0.91 .

Data on other variables Information on sociodemographic and lifestyle characteristics, including educational level and total physical activity, was collected at baseline. Anthropometric measurements were undertaken using standardised procedures and allowed the calculation of BMI $\left(\mathrm{kg} / \mathrm{m}^{2}\right)$ and WHR. Physical activity was expressed as the usual daily energy expenditure in metabolic equivalent task (MET) hours per day.

Study participants and follow-up From the original cohort of 28,572 participants, 1,027 were excluded because they could not be traced or did not respond during follow-up, 2,302 due to prevalent diabetes at enrolment, and 2,604 due to prevalent cancer $(n=694)$ and/or cardiovascular diseases $(n=1,880)$ and/or stroke $(n=902)$. An additional 344 participants were excluded due to missing values of dietary variables. The final sample of this analysis consisted of 22,295 individuals.

Active follow-up by specially trained health professionals was implemented at regular times by means of telephone interviews with the participants or, in the case of death, their next of kin.

Diabetes incidence We identified incident type 2 diabetes through indication of type 2 diabetes in medical records, discharge diagnosis or death certificates, first-reported type 2 diabetes-specific medication use or self-reported medical diagnosis of type 2 diabetes during follow-up. About $60 \%$ of all diabetic cases were verified by medical records. The date of diagnosis was set as the earliest date reported in medical records, discharge diagnosis or death certificates, if available, or the date reported in the questionnaire by the participant. If the exact date of diagnosis was missing and only the year of diagnosis was available, the date was set as 30 June of the indicated year.

Time to event was calculated until the date of diagnosis of diabetes, or the date of death from causes other than diabetes, or the date of the last contact prior to December 2011.

Statistical analysis All analyses were performed using Stata statistical software (version 11.0 for Windows; StataCorp LP, College Station, TX, USA). Frequency distributions for categorical variables and quartile values for continuous variables were estimated for descriptive purposes. Cox proportional hazards regression models were used to assess the relationships of diabetes with MDS (in categories 0-3, 4, 5, 6-9, as well as per two point increase), GL (in sex-specific quartiles, as well as per ten point increase) or with a combination of GL and MDS (GL $\leq$ or $>$ sex-specific median and MDS $\leq$ or $>$ median, 4). Models were adjusted for age $(<35$, 35-39, 40-44, 45-49, 50-54, 55-59, 60-64, 65-69, $\geq 70$ years, categorically), sex, level of education (four levels, categorically), BMI (sex-specific quintiles, categorically), physical activity expressed in MET h/day (sex-specific quintiles, categorically) and WHR (sex-specific quintiles, categorically). Models for MDS and for the combination of GL and MDS were also adjusted for total energy intake (sex-specific quintiles, categorically); those for GL, for non-carbohydrate energy intake (sex-specific quintiles, categorically), in order to avoid possible over-adjustment due to the high correlation between GL and carbohydrates (since carbohydrate intake contributes to the GL computation).

We also estimated HRs for diabetes according to GI (in sexspecific quartiles) through the model used for GL adjusting for total energy intake.

If the value of the adjustment variable was missing, we replaced it using the central category. Analyses were performed for the overall study population and in strata of age, sex, BMI and physical activity.

\section{Results}

During 234,935 person-years of follow-up (median follow-up 11.34 years), 2,330 cases of incident type 2 diabetes were recorded. Table 1 shows the baseline characteristics of the study participants according to MDS category and quartiles of GL (cut-offs for GL quartiles were 105.1, 129.0 and 158.0 for men and 87.0, 105.5 and 127.1 for women). Better educated participants tended to be more compliant with the Mediterranean diet. Younger participants reported a diet with a higher GL in comparison with older participants. GL correlated positively, but weakly, with MDS, with medians of 102.1, 111.4, 118.4 and 129.4 in successive MDS categories (Spearman's correlation coefficient 0.28).

HRs and corresponding 95\% CIs for incident type 2 diabetes in relation to MDS, overall and by BMI level, are shown in Table 2. Overall, a significant inverse association of increased adherence to the Mediterranean diet with type 2 diabetes emerged, with an HR of 0.88 (95\% CI $0.78,0.99)$ for MDS $\geq 6$ compared with MDS $\leq 3$. The corresponding HRs were $0.94(95 \%$ CI $0.63,1.40)$ for BMI $<25 \mathrm{~kg} / \mathrm{m}^{2}$ and 0.87 $\left(95 \%\right.$ CI $0.77,0.98$ ) for BMI $\geq 25 \mathrm{~kg} / \mathrm{m}^{2}$ ( $p$ for heterogeneity $0.868)$. No heterogeneity was evident by age $(p=0.560)$, sex $(p=0.487)$ or physical activity level $(p=0.495)$. 


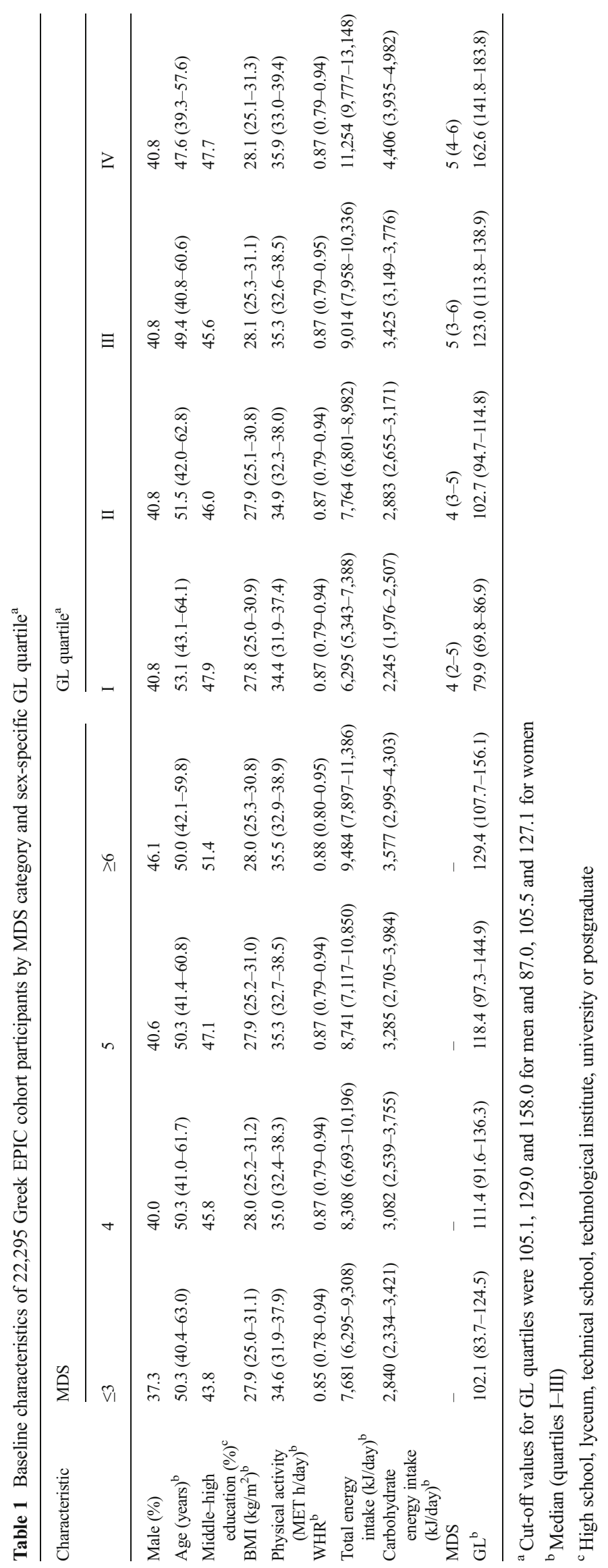


Table $2 \mathrm{HRs}^{\mathrm{a}}$ and $95 \%$ CIs for incident type 2 diabetes according to MDS category, overall and by strata of BMI

\begin{tabular}{|c|c|c|c|c|c|c|c|}
\hline \multirow[t]{2}{*}{ Variable } & \multicolumn{4}{|l|}{ MDS } & \multirow[t]{2}{*}{$\chi^{2}$ trend } & \multirow[t]{2}{*}{$p$ value } & \multirow[t]{2}{*}{ Two point increase } \\
\hline & $\leq 3$ & 4 & 5 & $\geq 6$ & & & \\
\hline \multicolumn{8}{|l|}{ Overall } \\
\hline Person-years & 73,977 & 50,925 & 50,391 & 59,542 & & & \\
\hline Cases $(n)$ & 716 & 529 & 503 & 582 & & & \\
\hline HR $(95 \% \mathrm{CI})$ & $1^{\mathrm{b}}$ & $1.00(0.89,1.12)$ & $0.94(0.84,1.06)$ & $0.88(0.78,0.99)$ & 5.33 & 0.021 & $0.94(0.89,0.99)$ \\
\hline \multicolumn{8}{|l|}{$\mathrm{BMI}^{\mathrm{c}}<25 \mathrm{~kg} / \mathrm{m}^{2}$} \\
\hline Person-years & 19,588 & 12,431 & 12,479 & 13,655 & & & \\
\hline Cases $(n)$ & 64 & 39 & 41 & 46 & & & \\
\hline HR $(95 \% \mathrm{CI})$ & $1^{\mathrm{b}}$ & $0.91(0.61,1.36)$ & $0.92(0.62,1.38)$ & $0.94(0.63,1.40)$ & 0.09 & 0.761 & $0.95(0.80,1.14)$ \\
\hline \multicolumn{8}{|l|}{$\mathrm{BMI}^{\mathrm{c}} \geq 25 \mathrm{~kg} / \mathrm{m}^{2}$} \\
\hline Person-years & & & & & & & \\
\hline Cases $(n)$ & 648 & 489 & 453 & 532 & & & \\
\hline HR (95\% CI) & $1^{\mathrm{b}}$ & $1.01(0.90,1.14)$ & $0.94(0.83,1.05)$ & $0.87(0.77,0.98)$ & 6.05 & 0.014 & $0.93(0.89,0.99)$ \\
\hline
\end{tabular}

${ }^{\text {a }}$ Proportional hazards regression models were adjusted for age, sex, education, BMI, physical activity, WHR and total energy intake

${ }^{\mathrm{b}}$ Reference category

${ }^{\mathrm{c}}$ The sum does not add up to the total because of some missing values

Table 3 presents the relation between type 2 diabetes and GL intake. The HRs for successive GL quartiles, compared with the lowest quartile of intake, were 1.11 (95\% CI 0.98, $1.25), 1.13(95 \%$ CI $0.99,1.29)$ and $1.21(95 \%$ CI $1.05,1.40)$, respectively, with a significant trend in risk $(p=0.013)$. Further adjustment for other dietary factors did not materially change the results. In particular, the HRs for the highest vs the lowest GL quartiles were $1.21(95 \%$ CI 1.05, 1.41) after adjustment for fats, $1.21(95 \%$ CI $1.02,1.44)$ after adjustment for fibre, $1.18(95 \%$ CI $1.02,1.37)$ after adjustment for alcohol and $1.20(95 \%$ CI $1.04,1.39)$ after adjustment for meat. The HR for the highest quartile of GL was 0.77 (95\% CI 0.47 , $1.25 ; p$ for trend 0.308 ) among participants with BMI $<25 \mathrm{~kg} / \mathrm{m}^{2}$ and $1.26(95 \%$ CI $1.08,1.47 ; p$ for trend 0.004 ) among those with BMI $\geq 25 \mathrm{~kg} / \mathrm{m}^{2}$ ( $p$ for heterogeneity $0.494)$. Results did not significantly differ by age $(p=0.113)$, sex $(p=0.951)$ or physical activity level $(p=0.415)$.

With reference to GI, compared with the lowest quartile, the HR was $1.14(95 \%$ CI 1.01, 1.29) for the second quartile, $1.13(95 \%$ CI $1.00,1.28)$ for the third quartile and $1.14(95 \%$

Table $3 \mathrm{HRs}^{\mathrm{a}}$ and $95 \%$ CIs for incident type 2 diabetes according to GL quartiles, overall and by strata of BMI

\begin{tabular}{|c|c|c|c|c|c|c|c|}
\hline \multirow[t]{2}{*}{ Variable } & \multicolumn{4}{|c|}{ GL quartile ${ }^{\mathrm{b}}$} & \multirow[t]{2}{*}{$\chi^{2}$ trend } & \multirow[t]{2}{*}{$p$ value } & \multirow[t]{2}{*}{ Ten point increase } \\
\hline & I & II & III & IV & & & \\
\hline \multicolumn{8}{|l|}{ Overall } \\
\hline Person-years & 59,234 & 58,885 & 59,073 & 57,743 & & & \\
\hline Cases $(n)$ & 539 & 592 & 606 & 593 & & & \\
\hline $\operatorname{HR}(95 \% \mathrm{CI})$ & $1^{\mathrm{c}}$ & $1.11(0.98,1.25)$ & $1.13(0.99,1.29)$ & $1.21(1.05,1.40)$ & 6.13 & 0.013 & $1.03(1.01,1.04)$ \\
\hline \multicolumn{8}{|c|}{$\mathrm{BMI}^{\mathrm{d}}<25\left(\mathrm{~kg} / \mathrm{m}^{2}\right)$} \\
\hline Person-years & 15,332 & 14,649 & 13,969 & 14,203 & & & \\
\hline Cases $(n)$ & 59 & 43 & 43 & 45 & & & \\
\hline $\operatorname{HR}(95 \% \mathrm{CI})$ & $1^{\mathrm{c}}$ & $0.79(0.53,1.20)$ & $0.80(0.52,1.26)$ & $0.77(0.47,1.25)$ & 1.04 & 0.308 & $0.99(0.95,1.05)$ \\
\hline \multicolumn{8}{|l|}{$\mathrm{BMI}^{\mathrm{d}} \geq 25\left(\mathrm{~kg} / \mathrm{m}^{2}\right)$} \\
\hline Person-years & 43,575 & 43,956 & 44,863 & 43,241 & & & \\
\hline Cases $(n)$ & 475 & 542 & 561 & 544 & & & \\
\hline $\mathrm{HR}(95 \% \mathrm{CI})$ & $1^{\mathrm{c}}$ & $1.14(1.00,1.30)$ & $1.18(1.03,1.35)$ & $1.26(1.08,1.47)$ & 8.38 & 0.004 & $1.03(1.01,1.04)$ \\
\hline
\end{tabular}

${ }^{\text {a }}$ Proportional hazards regression models were adjusted for age, sex, education, BMI, physical activity, WHR and total non-carbohydrate energy intake

${ }^{\mathrm{b}}$ Sex-specific quartiles

${ }^{\mathrm{c}}$ Reference category

${ }^{\mathrm{d}}$ The sum does not add up to the total because of some missing values 
CI 1.01, 1.30) for the highest quartile (ESM Table 2). There was no heterogeneity among strata of BMI $(p=0.772)$.

The combined effect of GL and MDS on the risk of incident type 2 diabetes is presented in Table 4. Compared with participants with a diet characterised by a high GL and a low MDS (i.e. GL >129.0/105.5 for men/women and MDS $\leq 4)$, the HRs were $0.89(95 \%$ CI $0.79,1.00)$ for those with a high GL diet and a high MDS; 0.89 (95\% CI $0.78,1.02)$ for those with a low GL diet and a low MDS; and 0.82 (95\% CI $0.71,0.95)$ for those with a low GL diet and a high MDS. The test for interaction was not significant $(p=0.722)$.

\section{Discussion}

These data indicate that both the Mediterranean diet and a low GL/GI diet were associated with a lower diabetes risk. The influence of the Mediterranean diet against diabetes risk was independent of GL levels ( $p$ for interaction 0.722), and individuals with a high MDS and a low GL tended to have the lowest diabetes risk. It is not difficult to envisage a low GL Mediterranean diet, since olive oil and vegetables dominate this diet and do not contribute, or contribute only marginally, to the GL. In this study, the combined protection imparted by a diet with a high MDS and a low GL was about $20 \%$.

A simple score assessing adherence to the Mediterranean diet was introduced by Trichopoulou and colleagues [26, 31] in 1995 and has since been used, with some variations, to evaluate the relation of the Mediterranean diet to overall mortality $[26,32,33]$ as well as to specific health outcomes including cardiovascular and cerebrovascular diseases [34, $35]$, overall cancer [22, 36-38] and various forms of cancer [39-41]. Type 2 diabetes shares several risk factors with these

Table $4 \mathrm{HRs}^{\mathrm{a}}$ and $95 \%$ CIs for incident type 2 diabetes according to GL and MDS

\begin{tabular}{lll}
\hline GL & \multicolumn{2}{l}{ MDS } \\
\cline { 2 - 3 } & $\begin{array}{l}\text { Low } \\
\leq 4\end{array}$ & $\begin{array}{l}\text { High } \\
>4\end{array}$ \\
\hline High $^{\mathrm{b}}$ & & \\
Diabetes incidence $^{\mathrm{c}}$ & $511 / 49,253$ & $688 / 67,563$ \\
HR $(95 \% \mathrm{CI})$ & $1^{\mathrm{d}}$ & $0.89(0.79,1.00)$ \\
Low $^{\mathrm{b}}$ & & \\
Diabetes incidence $^{\mathrm{c}}$ & $734 / 75,649$ & $397 / 42,470$ \\
HR $(95 \% \mathrm{CI})$ & $0.89(0.78,1.02)$ & $0.82(0.71,0.95)$ \\
\hline
\end{tabular}

${ }^{a}$ Proportional hazards regression models were adjusted for age, sex, education, BMI, physical activity, WHR and total energy intake

${ }^{\mathrm{b}}$ High GL > 129.0/105.5 for men/women; low GL $\leq 129.0 / 105.5$ for men/ women (i.e. sex-specific median)

${ }^{\mathrm{c}}$ Number of incident type 2 diabetes cases/person-years

${ }^{\mathrm{d}}$ Reference category conditions and has been inversely related to a closer conformity to the Mediterranean diet in various cohort studies [5-7]. In the largest one, which was based on 11,994 incident cases of diabetes from the EPIC cohort (not including data from Norway and Greece), the HRs for individuals with medium and high adherence to the Mediterranean diet were 0.93 (95\% CI $0.86,1.01)$ and 0.88 (95\% CI $0.79,0.97)$, respectively, compared with those with low adherence to the Mediterranean diet [7]. In that study, the association was attenuated in obese participants, a finding which was not evident in our investigation. The role of the Mediterranean diet in weight control remains controversial [42-44], and in most studies from Mediterranean countries the adherence to the Mediterranean diet was unrelated to overweight $[42,43]$. This suggests that the protection of the Mediterranean diet against diabetes is not through weight control but through several dietary characteristics of the Mediterranean diet. This issue is difficult to address, however, in cohort studies because information on weight changes during follow-up is rarely recorded.

In another cohort study, 13,380 Spanish university graduates were followed for a median of 4.4 years during which there were 103 incident cases of diabetes. Participants who closely adhered to a Mediterranean diet (MDS $\geq 7$ ) had a strikingly lower risk of diabetes (incidence rate ratio 0.17 ; $95 \%$ CI $0.04,0.72$ ) compared with those with the lowest adherence score (MDS $<3$ ) [5].

In the GISSI-Prevenzione study, which included 8,291 Italian patients with a recent myocardial infarction who were followed up for 3.5 years, a Mediterranean diet conveyed protection against incident diabetes (OR 0.65; 95\% CI 0.49, 0.85 ; highest quintile vs lowest quintile of adherence) [6]. In that study, however, the MDS was based on consumption of four foods only: cooked and raw vegetables, fruit, fish and olive oil [6]. Moreover, type 2 diabetic patients allocated to a Mediterranean diet may have better glycaemic control compared with diabetic patients following a control diet, as has been reported in various randomised trials [45].

It is unclear which component of the Mediterranean diet mostly contributes to its favourable effect. A particular feature of the Mediterranean diet is the use of extra-virgin olive oil, which leads to a high ratio of monounsaturated to saturated fatty acids. A review of dietary fat and diabetes suggests that replacing saturated and trans fats with unsaturated fats has beneficial effects on insulin sensitivity and is likely to reduce the risk of type 2 diabetes [46]. In a randomised trial, however, of individuals at high cardiovascular risk who were assigned to a Mediterranean diet supplemented with either free extravirgin olive oil or nuts and compared with individuals on a low-fat diet (comparison group), there was no difference in diabetes occurrence between the two variants of the Mediterranean diet when compared with the comparison group (HR 0.49 [95\% CI $0.25,0.97$ ] and HR 0.48 [95\% CI 0.24, 0.96], respectively) [8]. That study, however, had a relatively small 
sample size, consisting of 418 participants followed up for a median of 4 years with 54 incident cases of diabetes.

Our results on GL are in agreement with a systematic review published in 2008 that found a pooled RR of diabetes of $1.27(95 \%$ CI $1.12,1.45)$ for the highest vs the lowest GL quantiles [13]. Subsequently, eight more studies have examined this issue [14-21]. The largest one [14], based on an update of the Nurses' Health Study [10] and including almost 7,000 cases of incident diabetes, found a significant $30 \%$ increase in diabetes risk for the highest vs the lowest GL quintiles. Significant positive associations of similar magnitude emerged also from two other cohort studies from China [15] and the Netherlands [16], including about 1,600 and 900 incident cases, respectively. The other five studies found no clear associations [17-21]. All of them, however, were based on a relatively small number of cases, with the exception of the US Black Women's Health Study, which included more than 1,900 cases [19].

As in most previous works [13], we also found an association between diabetes and GI as an indicator of the glycaemic response to carbohydrates in different foods.

A high GL diet leads to rapid rises in blood glucose and insulin levels. The chronically increased insulin demand may eventually result in pancreatic beta cell failure and, as a consequence, impaired glucose tolerance and increased insulin resistance, which is a predictor of diabetes. A high dietary GL has also been unfavourably related to glycaemic control in individuals with diabetes [27].

Overweight individuals are generally more prone to uncontrolled postprandial hyperglycaemia after a glucose challenge. In two previous studies $[15,47]$, a stronger effect of high dietary GL on the development of diabetes in overweight and obese individuals was reported. Our results are not incompatible with this observation, although we did not find a statistically significant heterogeneity by BMI.

A strength of the study is that the cohort is based on the general population and involves a substantial fraction who still adhere to the traditional Mediterranean diet [48]. Moreover, the large sample size and the relatively long follow-up allowed the identification of a large number of incident diabetes cases. The prospective nature of the study, besides ensuring the temporal relation between exposure and disease, minimises the likelihood of information bias related to dietary habits recorded after disease occurrence. Since, however, this is an ongoing study and diet is a modifiable factor, we cannot be sure that the dietary habits of the cohort as recorded at baseline were consistent throughout the follow-up period. Nevertheless, we have excluded individuals with prevalent cancer, stroke or cardiovascular diseases, since they tend to modify their diet after diagnosis. In order to rule out a possible effect of subclinical diabetes on dietary habits, we repeated the analyses with a lag of 1 year and found essentially identical results.
It is known that a portion of the middle-aged and elderly population suffer from undiagnosed type 2 diabetes and, in our study, these people would be classified in the same group as those without diabetes. If, however, the dietary associations were similar for undiagnosed diabetic individuals as for diagnosed diabetic individuals this would tend to create a misclassification that could not exaggerate the underlying true association.

As far as confounding is concerned, overweight and obesity are the major risk factors for diabetes [2], and a high GL diet has been suggested to promote weight gain [49] (although the issue is open to discussion [50]). We took great care, however, to adjust for major factors related to body size, including both BMI and WHR, as well as energy intake and physical activity.

In conclusion, in a general population prospective cohort study, we found that a low GL diet that also adequately adheres to the principles of the traditional Mediterranean diet may reduce the incidence of type 2 diabetes mellitus.

Acknowledgements We thank I. Garimoldi (Department of Epidemiology, IRCCS - Istituto di Ricerche Farmacologiche Mario Negri, Milan, Italy) for her editorial assistance.

Funding The research leading to these results has received funding from the European Community's Seventh Framework Programme (FP7/ 2007-2013) under CHANCES Project grant agreement no. HEALTHF3-2010-242244. The work of CLV, MR and FT was partially supported by the Italian Association for Cancer Research (AIRC), Milan, Italy (grant no.10068).

Duality of interest The authors declare that there is no duality of interest associated with this manuscript.

Contribution statement MR and FT analysed the data and drafted the paper. PL, DT, CLV and AT contributed to the conception and design of study and acquisition of data. LSA contributed to the interpretation of data. All authors contributed to the critical revision of the manuscript and approved the final version. CLV and AT share primary responsibility for the final content.

\section{References}

1. Yach D, Stuckler D, Brownell KD (2006) Epidemiologic and economic consequences of the global epidemics of obesity and diabetes. Nat Med 12:62-66

2. Hu FB, Manson JE, Stampfer MJ et al (2001) Diet, lifestyle, and the risk of type 2 diabetes mellitus in women. N Engl J Med 345:790 797

3. Montonen J, Knekt P, Harkanen T et al (2005) Dietary patterns and the incidence of type 2 diabetes. Am J Epidemiol 161:219-227

4. Fung TT, Schulze M, Manson JE, Willett WC, Hu FB (2004) Dietary patterns, meat intake, and the risk of type 2 diabetes in women. Arch Intern Med 164:2235-2240

5. Martinez-Gonzalez MA, de la Fuente-Arrillaga C, Nunez-Cordoba JM et al (2008) Adherence to Mediterranean diet and risk of developing diabetes: prospective cohort study. BMJ 336:1348-1351 
6. Mozaffarian D, Marfisi R, Levantesi G et al (2007) Incidence of newonset diabetes and impaired fasting glucose in patients with recent myocardial infarction and the effect of clinical and lifestyle risk factors. Lancet 370:667-675

7. Romaguera D, Guevara M, Norat T et al (2011) Mediterranean diet and type 2 diabetes risk in the European Prospective Investigation into Cancer and Nutrition (EPIC) study: the InterAct project. Diabetes Care 34:1913-1918

8. Salas-Salvado J, Bullo M, Babio N et al (2011) Reduction in the incidence of type 2 diabetes with the Mediterranean diet: results of the PREDIMED-Reus nutrition intervention randomized trial. Diabetes Care 34:14-19

9. Jenkins DJ, Wolever TM, Taylor RH et al (1981) Glycemic index of foods: a physiological basis for carbohydrate exchange. Am J Clin Nutr 34:362-366

10. Salmeron J, Manson JE, Stampfer MJ, Colditz GA, Wing AL, Willett WC (1997) Dietary fiber, glycemic load, and risk of noninsulin-dependent diabetes mellitus in women. JAMA 277:472-477

11. Salmeron J, Ascherio A, Rimm EB et al (1997) Dietary fiber, glycemic load, and risk of NIDDM in men. Diabetes Care 20:545-550

12. Augustin LS, Franceschi S, Jenkins DJ, Kendall CW, La Vecchia C (2002) Glycemic index in chronic disease: a review. Eur J Clin Nutr 56:1049-1071

13. Barclay AW, Petocz P, McMillan-Price J et al (2008) Glycemic index, glycemic load, and chronic disease risk - a meta-analysis of observational studies. Am J Clin Nutr 87:627-637

14. Mekary RA, Rimm EB, Giovannucci E et al (2011) Joint association of glycemic load and alcohol intake with type 2 diabetes incidence in women. Am J Clin Nutr 94:1525-1532

15. Villegas R, Liu S, Gao YT et al (2007) Prospective study of dietary carbohydrates, glycemic index, glycemic load, and incidence of type 2 diabetes mellitus in middle-aged Chinese women. Arch Intern Med 167:2310-2316

16. Sluijs I, van der Schouw YT, van der A DL (2010) Carbohydrate quantity and quality and risk of type 2 diabetes in the European Prospective Investigation into Cancer and Nutrition-Netherlands (EPIC-NL) study. Am J Clin Nutr 92:905-911

17. Mosdol A, Witte DR, Frost G, Marmot MG, Brunner EJ (2007) Dietary glycemic index and glycemic load are associated with highdensity-lipoprotein cholesterol at baseline but not with increased risk of diabetes in the Whitehall II study. Am J Clin Nutr 86:988-994

18. Sahyoun NR, Anderson AL, Tylavsky FA, Lee JS, Sellmeyer DE, Harris TB (2008) Dietary glycemic index and glycemic load and the risk of type 2 diabetes in older adults. Am J Clin Nutr 87:126-131

19. Krishnan S, Rosenberg L, Singer M et al (2007) Glycemic index, glycemic load, and cereal fiber intake and risk of type 2 diabetes in US black women. Arch Intern Med 167:2304-2309

20. van Woudenbergh GJ, Kuijsten A, Sijbrands EJ, Hofman A, Witteman JC, Feskens EJ (2011) Glycemic index and glycemic load and their association with C-reactive protein and incident type 2 diabetes. J Nutr Metab. doi:10.1155/2011/623076

21. Sakurai M, Nakamura K, Miura K et al (2012) Dietary glycemic index and risk of type 2 diabetes mellitus in middle-aged Japanese men. Metabolism 61:47-55

22. Benetou V, Trichopoulou A, Orfanos P et al (2008) Conformity to traditional Mediterranean diet and cancer incidence: the Greek EPIC cohort. Br J Cancer 99:191-195

23. Riboli E, Hunt KJ, Slimani N et al (2002) European Prospective Investigation into Cancer and Nutrition (EPIC): study populations and data collection. Public Health Nutr 5:1113-1124

24. Gnardellis C, Trichopoulou A, Katsouyanni K, Polychronopoulos E, Rimm EB, Trichopoulos D (1995) Reproducibility and validity of an extensive semiquantitative food frequency questionnaire among Greek school teachers. Epidemiology 6:74-77

25. Trichopoulou A, Georga K (2004) Composition tables of foods and Greek dishes. Parisianos, Athens
26. Trichopoulou A, Costacou T, Bamia C, Trichopoulos D (2003) Adherence to a Mediterranean diet and survival in a Greek population. N Engl J Med 348:2599-2608

27. Wolever TM, Nguyen PM, Chiasson JL et al (1994) Determinants of diet glycemic index calculated retrospectively from diet records of 342 individuals with non-insulin-dependent diabetes mellitus. Am J Clin Nutr 59:1265-1269

28. Foster-Powell K, Holt SH, Brand-Miller JC (2002) International table of glycemic index and glycemic load values: 2002. Am J Clin Nutr 76:5-56

29. van Bakel MM, Kaaks R, Feskens EJ et al (2009) Dietary glycaemic index and glycaemic load in the European Prospective Investigation into Cancer and Nutrition. Eur J Clin Nutr 63(Suppl 4):S188-S205

30. Henry CJ, Lightowler HJ, Strik CM, Storey M (2005) Glycaemic index values for commercially available potatoes in Great Britain. Br J Nutr 94:917-921

31. Trichopoulou A, Kouris-Blazos A, Wahlqvist ML et al (1995) Diet and overall survival in elderly people. BMJ 311:1457-1460

32. Sofi F, Cesari F, Abbate R, Gensini GF, Casini A (2008) Adherence to Mediterranean diet and health status: meta-analysis. BMJ 337:a1344

33. Lagiou P, Trichopoulos D, Sandin S et al (2006) Mediterranean dietary pattern and mortality among young women: a cohort study in Sweden. Br J Nutr 96:384-392

34. Misirli G, Benetou V, Lagiou P, Bamia C, Trichopoulos D, Trichopoulou A (2012) Relation of the traditional Mediterranean diet to cerebrovascular disease in a Mediterranean population. Am J Epidemiol 176:1185-1192

35. Estruch R, Ros E, Salas-Salvado J et al (2013) Primary prevention of cardiovascular disease with a Mediterranean diet. N Engl J Med 368:1279-1290

36. Trichopoulou A, Lagiou P, Kuper H, Trichopoulos D (2000) Cancer and Mediterranean dietary traditions. Cancer Epidemiol Biomarkers Prev 9:869-873

37. La Vecchia C, Bosetti C (2006) Diet and cancer risk in Mediterranean countries: open issues. Public Health Nutr 9:1077-1082

38. Pelucchi C, Bosetti C, Rossi M, Negri E, La Vecchia C (2009) Selected aspects of Mediterranean diet and cancer risk. Nutr Cancer 61:756-766

39. Bosetti C, Gallus S, Trichopoulou A et al (2003) Influence of the Mediterranean diet on the risk of cancers of the upper aerodigestive tract. Cancer Epidemiol Biomarkers Prev 12:1091-1094

40. Trichopoulou A, Bamia C, Lagiou P, Trichopoulos D (2010) Conformity to traditional Mediterranean diet and breast cancer risk in the Greek EPIC (European Prospective Investigation into Cancer and Nutrition) cohort. Am J Clin Nutr 92:620-625

41. Buckland G, Agudo A, Lujan L et al (2010) Adherence to a Mediterranean diet and risk of gastric adenocarcinoma within the European Prospective Investigation into Cancer and Nutrition (EPIC) cohort study. Am J Clin Nutr 91:381-390

42. Rossi M, Negri E, Bosetti C et al (2008) Mediterranean diet in relation to body mass index and waist-to-hip ratio. Public Health Nutr 11:214-217

43. Trichopoulou A, Naska A, Orfanos P, Trichopoulos D (2005) Mediterranean diet in relation to body mass index and waist-to-hip ratio: the Greek European Prospective Investigation into Cancer and Nutrition Study. Am J Clin Nutr 82:935-940

44. Romaguera D, Norat T, Vergnaud AC et al (2010) Mediterranean dietary patterns and prospective weight change in participants of the EPIC-PANACEA project. Am J Clin Nutr 92:912-921

45. Esposito K, Maiorino MI, Ceriello A, Giugliano D (2010) Prevention and control of type 2 diabetes by Mediterranean diet: a systematic review. Diabetes Res Clin Pract 89:97-102

46. Riserus U, Willett WC, Hu FB (2009) Dietary fats and prevention of type 2 diabetes. Prog Lipid Res 48:44-51

47. Hodge AM, English DR, O'Dea K, Giles GG (2004) Glycemic index and dietary fiber and the risk of type 2 diabetes. Diabetes Care 27:2701-2706 
48. DAFNE Data Food Networking (2008) The DAFNE initiative. Available from www.nut.uoa.gr/dafnesoftweb. Accessed 28 June 2013

49. Maki KC, Rains TM, Kaden VN, Raneri KR, Davidson MH (2007) Effects of a reduced-glycemic-load diet on body weight, body composition, and cardiovascular disease risk markers in overweight and obese adults. Am J Clin Nutr 85:724-734

50. Rossi M, Bosetti C, Talamini R et al (2010) Glycemic index and glycemic load in relation to body mass index and waist to hip ratio. Eur J Nutr 49:459-464 